\title{
Anaesthesia Department
}

\section{Preparedness and Response for the COVID-19 Outbreak in Portugal: A Perspective from CUF Porto Hospital}

\section{Preparação e Resposta do Departamento de Anestesia para o Surto COVID-19 em Portugal: Uma Perspetiva do Hospital CUF Porto}

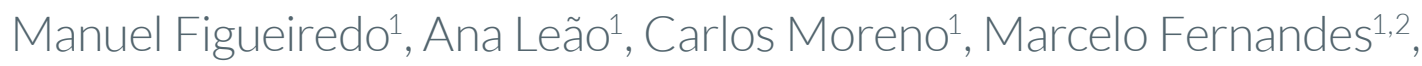
Angel Hernandez $z^{1}$, José Manuel Correia ${ }^{1,3}$, Marcos Gouveia ${ }^{1}$, Paulo Ramos ${ }^{1,4}$, Sara Fonseca ${ }^{1,4}$, Tiago Faria ${ }^{1,2}$, Pedro Santos ${ }^{1}$, Raquel Oliveira ${ }^{1,4}$, Sara Gomes ${ }^{1,5}$

Corresponding Author/Autor Correspondente: Manuel Figueiredo [manuel.figueiredo@jmellosaude.pt] Estrada da Circunvalação 14341, 4100-180 Porto, Portugal

KEYWORDS: Coronavirus Infections; COVID-19; Disease Outbreaks; Operating Rooms; Pandemics PALAVRAS-CHAVE: Bloco Operatório; COVID-19; Infeções por Coronavírus; Pandemia; Surtos de Doenças

\section{INTRODUCTION}

A global health emergency has been declared by the World Health Organization (WHO) as the SARS-CoV-2 outbreak spread across the world. Patients infected with SARS-CoV-2 are at risk for developing respiratory failure and requiring admission to critical care units. While providing optimal treatment for these patients, careful execution of infection control measures is necessary to prevent nosocomial transmission to other patients and to health workers providing care. ${ }^{1}$ In the operating room, these preparations involve multiple stakeholders and can present a significant challenge. Here we described the measures for the outbreak adopted by the anesthesia department of a private tertiary care level hospital in Porto. These include engineering controls such as: identification and preparation of an isolation operating rooms, administrative measures such as: modification of workflow and processes, introduction of personal protective equipment, and formulation of clinical guidelines for anaesthetic management. We discuss how the hierarchy of controls should be a framework to plan, the necessary measures during each phase of a pandemic, and review the evidence of the procedures taken. The-

1. Department of Anaesthesia, CUF Porto Hospital, Porto, Portugal. 2. Department of Anaesthesia, Hospital Braga, Braga, Portugal. 3. Burn Unit, Centro Hospitalar e Universitário São João, Porto, Portugal. 4. Department of Anaesthesia, Centro Hospitalar e Universitário São João, Porto, Portugal. 5. School of Medicine, Universidade Minho, Braga, Portugal.

- Author(s) (or their employer(s)) 2020. Re-use permitted under CC BY-NC. No commercial re-use. ${ }^{\bullet}$ Autor (es) (ou seu (s) empregador (es)) 2020. Reutilização permitida de acordo com CC BY-NC. Nenhuma reutilização comercial. 
se containment measures are necessary to optimize the quality of care provided to SARS-CoV-2 patients and to minimize the risk of viral transmission to other patients or healthcare workers. ${ }^{2}$

\section{EMERGENCE AND GLOBAL SPREAD OF SARS-CoV-2}

Coronaviruses are important human and animal pathogens. At the end of 2019, a novel coronavirus was identified as the cause of a cluster of pneumonia cases in the city of Wuhan, in the Hubei Province of China. It rapidly spread, resulting in an epidemic throughout China, followed by an increasing number of cases in other countries throughout the world. In February 2020, the World Health Organization designated the disease

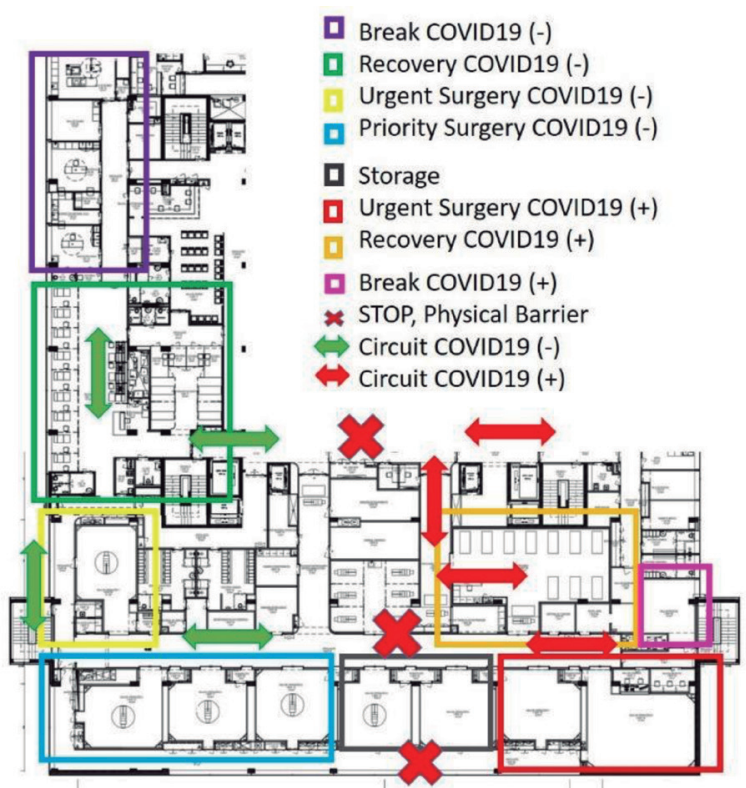

FIGURE 1. Operating rooms for positive and negative COVID pa tients.
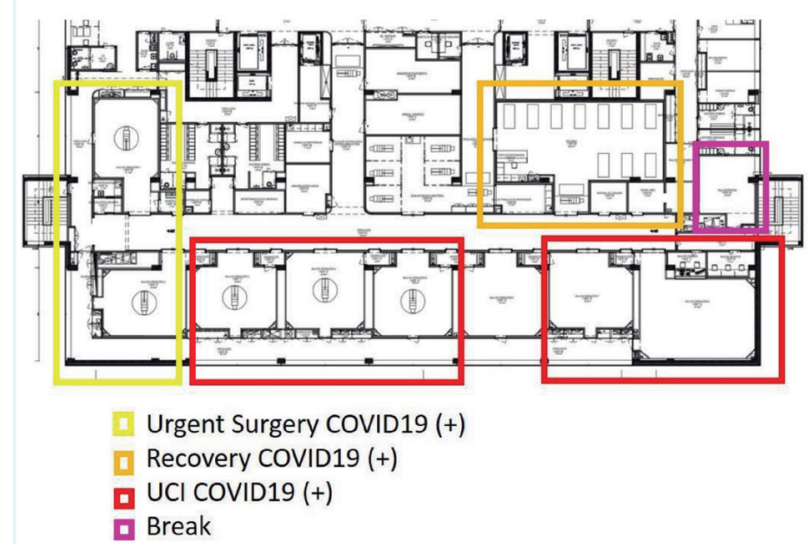

FIGURE 2. Operating theatre for ICU and surgery in positive COVID patients.
COVID-19, which stands for coronavirus disease 2019 that can evolve to a severe acute respiratory syndrome coronavirus 2 (SARS-CoV-2). ${ }^{2}$

In light of the worsening global situation, WHO declared the COVID-19 a pandemic on March 11, 2020. Healthcare systems worldwide needed to adapt to respond to Coronavirus Disease 2019 (COVID-19). Patients with COVID-19 can progress from asymptomatic or mild illness to hypoxemic respiratory failure or multisystem organ failure, requiring intubation and intensive care admission and management for a prolonged period. Healthcare providers are at the frontline of this epidemic, and they need to be aware of the best available evidence to guide therapeutic management of patients with COVID-19 and to keep themselves safe while doing so. ${ }^{3}$

Given that these patients may, in hospital admission by other clinical conditions or even in the evolution of their disease, need to enter an operating room, the role of the anaesthesiologist in optimizing the routes, measures and protocols is crucial.

The Anaesthesiology Department from CUF-Porto Hospital (HCP) developed disease outbreak response measures in coordination with surgical, nursing, and other allied health staff. The two main goals of these procedures were to favor the care of patients with knowh or suspected COVID-19 who needed surgery, minimizing the risk of perioperative viral transmission to health workers and other patients and to support every patient with a need for an emergency surgery. ${ }^{1}$

This article aims to describe the measures taken to address these goals, including identification and set-up of an isolation operating rooms (OR), modification of workflow and processes, management of staff, and formulation of clinical guidelines for the anaesthetic management.

\section{HOSPITAL MEASURES RELATED TO SURGERY AND ANAESTHESIA DURING THE COVID-19 OUTBREAK}

\section{INSTITUTIONAL GUIDELINES FOR PERIOPERATIVE MANAGEMENT}

\section{Operating Room Management}

Soon after the National Health Direction established that every patient considered non urgent/nonpriority should be postponed and discharged, CUF Porto hospital adapted their operating theatres in order to receive COVID-19 patients and comply with the highest safety 
standards for patients and workers. In fact, elective surgeries were limited to interventions in oncologic and obstetric surgery. Urgent operations continued, but with additional concerns regarding the risk of disease spread, namely implementing an increased turnaround time between surgeries, in order to accommodate additional infection prevention measures.

CUF Porto hospital has an operating theatre with 7 operating rooms fully equipped and different contingency plans were developed in accordance to distinct levels of demands.

\section{Plan A}

The Fig. 1 represents the distribution plan for operating room in case of positive/suspicious COVID-19 patients with indication for urgent surgery and negative COVID-19 patients with indication for urgent or priority surgery.

\section{Plan B}

This plan was ready to be implemented in the case of mitigation and the need for extra intensive care beds (Fig. 2), where 5 rooms were reserved for intensive care and 2 rooms for surgeries in COVID-19 patients.

\section{Plan C}

The Plan $C$ is nowadays in practice and defined two groups of operating rooms; two rooms for positive or suspicious COVID-19 patients with indication for urgent surgery and five rooms for negative COVID-19 patients with indication for urgent or elective surgery.

In the different plans, separate circuits to go and return from the operating theatre, were developed in order to isolate COVID-19 positive/suspicious patients from negative.

At CUF Porto Hospital, all the OR were already equipped with pressure systems able to create negative or positive environments, which may contribute to reducing aerosols contagion risk.

Once the emergency period was over, we assisted to a gradually returning to the normalization of surgical practice with the elective surgery restart. The operating theatre had to be restructured in order to allow the coexistence of positive and negative COVID-19 patients complying with isolated circuits (Fig. 3).

The operating rooms used for positive COVID-19 patients or aerosol-generating surgeries, such as bronchoscopy and dental treatments, were kept in negative pressure environment and the remaining rooms worked with positive pressure environment and 21 air exchanges per hour.
With this operating theatre contingency measures, it was possible to ensure the correct and safe different levels of demand created by the COVID-19 pandemic and above all to ensure our patients and staff safety contributing to the empowerment healthcare system as a whole.

\section{Staff Management, Training and communication}

The use of a surgical mask was made obligatory in all areas of the operating theatre as in all areas of the Hospital, for all hospital workers. Staff elements were monitored for fever and respiratory symptoms, but there was no record of any suspected/confirmed case in our operating room team.

Anaesthesiology members were involved in research and in the organization of cognitive tools such as protocols for personal protective equipment usage (PPE) and patient management.

Due to a reduced number of surgeries, nurses, anaesthesiologists and technical staff were able to attend in situ simulation training on donning and doffing, in a special area created for safe practice. These sessions were always monitored by a third element that could confirm the correct execution of the procedure. Everyday a new personal protective equipment was suitable for all healthcare teams in the operating room.

We established a straight communication channel between all the staff involved in patient management, including healthcare workers from the emergency department, intensive care unit, wards, labs and outpatient appointments. Before patient transference, a phone call from the head nurse to her correspondent at the place receiving the patient was done. In order to reduce the risk and even avoid contamination, the transport was done through the faster pathway with less care workers

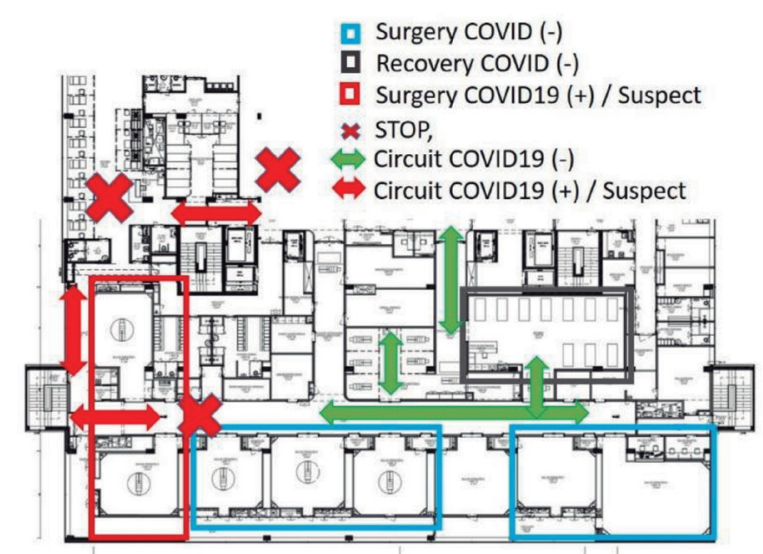

FIGURE 3. Discontent plan for operating theatre. 
nearby. After each patient's displacement a cleaning team was contacted by phone to clean the pathway and surfaces deeply.

\section{Patient Management}

\section{Admission}

All patients presenting to the hospital were screened using a questionnaire for suspected SARS-CoV-2 infection, which included questions about travel to endemic regions in the past 14 days, close contact with identified SARS-CoV-2 infected patients, fever or respiratory symptoms. After this clinical screening, the patient was tested with RT-PCR for SARS-CoV-2.

In cases of urgent surgical procedures, whenever possible, all patients were tested with RT-PCR for SARS-CoV-2. This procedure was also done with all pregnant in labor or proposed for cesarean-section section. In all women in labor that were positive/suspected or in the cases with no time to obtain test results, the delivery was done in an operative room with negative pressure ventilation and with full protective equipment for all staff members involved (Fig. 3).

\section{Anesthesia Care}

CUF Porto Hospital adopted the same strategy as previously described by many authors, namely recommendations concentrated in organization of the operating theatre; airway management and ventilation and teamwork. $^{4-7}$ The anaesthesia team was daily organized in shifts of 24 hours per week. Each day two consultants in anaesthesiology were in physical presence and another element was on-call.

Before the begin of anaesthesia care, a briefing was done by anaesthesiologist and anaesthesia nurse in order to discuss patient history and comorbidities; define the anaesthesia intervention, including airway approach, complementary procedures (such as arterial line or central venous access); and mainly to anticipate possible difficulties and plan for the solutions.

The team of one consultant in Anaesthesiology and one expertise anaesthesia nurse begin donning PPE under the observation of a third element (usually another nurse). This element also observes the donning of a rescue experienced nurse that will be adjacent to the operating room and perceives all anaesthesia and surgery procedures and helps if there is a need for new equipment or special assistance.

All the anaesthesia drugs and airway devices are previously prepared and organized in trays. We adopted the approach of a rapid induction with orotracheal in- tubation, using a videolaryngoscope with dischargeable blades (McGraph ${ }^{\mathrm{TM}}$ ) with a clamped tube. For protection of droplets and aerosolization a transparent plastic CoVering the patient's head, face and neck was used. This protection only was removed when the patient was completely awake and with his own face mask.

The reCoVery of anaesthesia was done inside the operating room and the patient was discharged to ward. In the case of a patient needing intensive care, after surgery, the patient and the anaesthesia team will stay for approximately 30 minutes inside the OR before transferring.

A proper place for doffing the PPE was placed nearby the corridor of the operating room and the procedure is done under mirror and peer visualization.

\section{Visitors}

All visitors to the hospital needed to be tested with RT-PCR. In cesarean sections, HCP did not allow the presence of any companion to the pregnant women during the surgery. In the case of children surgeries, the parents who wanted to enter with their relatives until the induction of anaesthesia (one parent per child), needed to be tested for COVID-19 in the previous 48 hours.

\section{INSTITUTIONAL GUIDELINES FOR ANESTHESIA PROCEDURES OUT OF THE OPERATING ROOM}

At CUF Porto hospital there are different areas where anaesthetic acts are performed outside the classic operating room. It was necessary to create contingency plans and later discontent plans appropriate to each area.

\section{Special Exams Unit:}

The procedures performed under anaesthesia in this area are digestive endoscopic examinations, flexible bronchoscopy, dental treatments, transesophageal echocardiograms and auditory evoked potentials.

The contingency plan applied in this area at the beginning of the pandemic in Portugal was:

- Cancellation of all elective exams, only urgent exams were performed;

- Organization of positive COVID/suspicious circuit and negative COVID circuit, from hospital admission to exams area, reCoVery, hospitalization if necessary and hospital discharge;

- In urgent procedures, the proposed patients should preferably have a SARS-CoV-2 test performed 4-6 hours before the procedure;

- If it was not possible to wait for the test result due to 
the urgency of the procedure, the case was assumed to be positive for SARS-CoV-2, following the defined positive COVID circuit. In this situation, the exams were performed in negative pressure rooms in the classic surgery operating room;

- Positive SARS-CoV-2 test patients were postponed if possible;

- Emergent and urgent positive COVID patients followed the established positive COVID circuit;

- All elements involved in the procedures (doctors, nurses, assistants) used appropriate PPE;

- Careful disinfection of the examination room and patient circuit were done.

The discontent plan applied in this area came later with the aim of restarting the non-urgent exams:

- Urgent and elective exams performed;

- Positive COVID/suspicious and negative COVID circuits were maintained from hospital admission to exams area, reCoVery room, hospitalization if necessary and hospital discharge;

- All patients proposed for elective procedures should have a negative SARS-CoV-2 test performed in the last 48 hours and should not have acute respiratory symptoms;

- In urgent procedures, the proposed patients should have a SARS-CoV-2 test performed, preferably between 4-6 before the procedure;

- If it was not possible to wait for the test result due to the urgency of the procedure, it is assumed to be a positive case for SARS-CoV-2, following the defined positive COVID circuit;

- Patients with a positive test should be postponed if possible:

- All elements involved in the procedures (doctors, nurses, assistants) use appropriate PPE;

- Careful disinfection of the examination room and patient circuit are done.

\section{Obstetrics and Gynecology Unit}

\section{Contingency plan:}

In the initial phase of the pandemic in Portugal, the contingency plan in this area was essentially based on:

- Cancellation of all non-urgent gynecological procedures;

- Only deliveries, cesarean sections and urgent oncological gynecological surgery were performed;
- Organization of positive COVID/suspicious and negative COVID circuits from hospital admission, hospitalization, operating room, reCoVery room, hospitalization and hospital discharge;

- Conducting tests for SARS-CoV-2 research 48 hours before elective surgery (deliveries, cesarean sections and urgent gynecology surgery);

- In urgent procedures, the proposed patients should have a SARS-CoV-2 test performed, preferably between 4-6 hours before surgery;

- If emergent surgery with the impossibility of carrying out the SARS-CoV-2 research test, the case was assumed to be positive COVID and the patient should follow the positive COVID circuit;

- Use of appropriate PPE by all elements involved in the surgeries (doctors, nurses and assistants);

- Careful disinfection of the operating room and the circuit traveled by the patient.

Discontent plan arises when non-urgent surgeries were restarted:

- Urgent and non-urgent gynecological surgery, deliveries and cesarean sections;

- Positive COVID/suspicious and negative COVID circuits were maintained, since hospital admission, hospitalization, operating theater, reCoVery room, hospitalization and hospital discharge;

- Conducting tests for SARS-CoV-2 research performed 48 hours before elective surgery;

- In urgent procedures, the proposed patients should have a SARS-CoV-2 test performed, preferably between 4-6 hours before surgery;

- If emergent surgery with the impossibility of carrying out the SARS-CoV-2 research test in a timely manner, the case was assumed to be positive and the patient followed the positive COVID circuit;

- Use of appropriate PPE by all elements involved in the surgeries (doctors, nurses and assistants);

- Careful disinfection of the operating room/delivery room and circuit traveled by the patient.

Hemodynamics and Intervention Radiology Unit

Contingency plan:

- Cancellation of all non-urgent procedures;

- Organization of positive COVID +/suspicious and negative COVID circuits from hospital admission, hemodynamics room, reCoVery room, hospitalization if necessary and hospital discharge; 
- In urgent procedures, the proposed patients should have a SARS-CoV-2 test performed preferably between 4-6 hours before surgery;

- If it was not possible to wait for the test result due to the urgency of the procedure, it was assumed that the patient was positive for SARS-CoV-2, and the patient must follow the defined positive COVID circuit;

- Patients with a positive test were, if possible, postponed;

- Emergent and urgent positive COVID patients followed the established positive COVID circuit;

- Use of appropriate PPE by all elements involved in the surgeries (doctors, nurses, and assistants);

- Careful disinfection of the operating room/delivery room and the circuit traveled by the patient.

\section{Discontent plan emerges when non-urgent procedures} were restarted:

- Urgent and elective procedures;

- The positive COVID/suspicious and negative COVID circuits were maintained, since hospital admission, hemodynamics room, reCoVery room, hospitalization if necessary and hospital discharge;

- Conducting tests for SARS-CoV-2 research 48 hours in advance for scheduled procedures;

- In urgent procedures, the proposed patients should have a SARS-CoV-2 test performed in a timely manner, preferably between 4-6 hours before the procedure;

- If an emergent procedure with the impossibility of carrying out the SARS-CoV-2 research test in a timely manner, the case was assumed to be positive and the patient must follow the defined positive COVID circuit;

- Use of appropriate PPE by all elements involved in the surgeries (doctors, nurses and assistants);

- Careful disinfection of the hemodynamics room and the circuit CoVered by the patient.

Nuclear Magnetic Resonance (NMR) and Computed Tomography (CT)

\section{Contingency plan:}

- Cancellation of all elective exams;

- Organization of positive COVID/suspicious and negative COVID circuits from hospital admission, exams room, reCoVery room and hospital discharge;
- In urgent procedures, the proposed patients should have a SARS-CoV-2 test performed in a timely manner, preferably between 4-6 hours;

- If it was not possible to wait for the test result due to the urgency of the procedure, it was assumed that the patient was suspected/positive for SARS-CoV-2, and must follow the defined positive COVID circuit;

- Patients with a positive test were, if possible, postponed;

- Emerging and urgent positive COVID patients followed the established positive COVID circuit;

- Use of appropriate PPE by all elements involved in the surgeries (doctors, nurses and assistants);

- Careful disinfection of the exams room and the circuit traveled by the patient.

Discontent plan emerges when non-urgent procedures were restarted:

- Urgent and non-urgent procedures;

- The positive COVID/suspicious and negative COVID circuits were maintained, circuits from hospital admission, exams room, reCoVery room and hospital discharge;

- Conducting tests for SARS-CoV-2 research 48 hours in advance for scheduled procedures;

- In urgent procedures, the proposed patients should have a SARS-CoV-2 test performed in a timely manner, preferably between 4-6 hours;

- If an emerging procedure with the impossibility of carrying out the SARS-COV-2 research test in a timely manner, the case was assumed to be positive following the positive COVID circuit;

- Use of appropriate PPE by all elements involved in the surgeries (doctors, nurses and assistants);

- Careful disinfection of the examination room and the circuit the patient travels.

\section{DISCUSSION}

The Anesthesiology Department of CUF Porto Hospital formed clinical care, infection control and manpower allocation workgroups at the beginning of the COVID-19 outbreak, in order to develop guidelines that were regularly updated for the clinical management of suspected or confirmed cases. ${ }^{6}$ Teamwork, good communication across all the departments and willingness to listen are key components to maintaining good patient care, especially in these times of crisis. 
One of the highlights of our experience during the outbreak was the temporary set-up of a negative-pressure operation room with an adequate pressure gradient ( $\geq 2.5 \mathrm{~Pa}$ ). There is likely to be a very limited number of hospitals that are prepared with negative-pressure operating rooms. ${ }^{8}$ Overall, although the risk of cross-contamination from airborne infection is low, if staff are adequately protected with appropriate personal protective equipment, a negative pressure operating theatre can offer optimal protection to personal working in adjacent areas.

Almost all hospitals generally have positive-pressure operating rooms and they may experience an outbreak without the facilities for the perioperative management of COVID-19 patients. Our hospital changed two operating rooms pressure according to the Centers for Disease Control and Prevention (CDC) guidelines. ' Continuous negative pressure was maintained in this two operating rooms (-4.7 $\mathrm{Pa}$ ) and reserved for procedures of suspected or confirmed COVID-19 patients, for aerosol-generating procedures such as bronchoscopy and dental treatments in any patient. The other operating rooms are maintained 21 air exchanges per hour, so we managed a well-ventilated operating theatre.

Personal protective equipment has become an important and sensitive subject during the current COVID-19 pandemic. However, is important to remember that PPE is only one part of a system to prevent contamination of those who are working near patients with COVID-19. It is vital to perform other procedures of a system to reduce cross-infection. In our hospital remains crucial that all staff understand the purpose of PPE and its role as one part of a complex system to reduce transmission from patients to staff and other patients. The PPE's setting used must be the appropriate to the mode of infection. Misuse of PPE depletes limited stocks, leads to avoidable shortages and increases the risk of staff. Therefore, when performing procedures that generate aerosols, such as endotracheal intubation in patients with COVID-19, health care workers must wear enhanced PPE, including gloves, a gown, either a face-shield that fully CoVers the front and sides of the face or goggles and respiratory protection, at least as protective as an N95 filtering face piece respirator. ${ }^{10}$ When removing

TABLE 1. Summary of contingency plan.

\begin{tabular}{|c|c|c|c|c|}
\hline & $\begin{array}{l}\text { SPECIAL EXAMS } \\
\text { UNIT }\end{array}$ & $\begin{array}{l}\text { OBSTETRICS AND } \\
\text { GYNECOLOGY UNIT }\end{array}$ & $\begin{array}{c}\text { HEMODYNAMICS AND } \\
\text { INTERVENTION RADIOLOGY UNIT }\end{array}$ & $\begin{array}{l}\text { NMR/ CT } \\
\text { UNIT }\end{array}$ \\
\hline ONLY URGENT PROCEDURES & $x$ & $\begin{array}{l}\text { Exception for elective } \\
\text { c-sections }\end{array}$ & $x$ & $x$ \\
\hline IF POSSIBLE, TEST BEFORE & $x$ & $x$ & $x$ & $x$ \\
\hline $\begin{array}{l}\text { URGENT, TEST IN 6H } \\
\text { BEFORE PROCEDURE }\end{array}$ & $x$ & $x$ & $x$ & $x$ \\
\hline EMERGENT SUCHAS COVID + & $x$ & $x$ & $x$ & $x$ \\
\hline PPE FOR ALL STAFF & $x$ & $x$ & $x$ & $x$ \\
\hline DISINFECTION & $x$ & $x$ & $x$ & $x$ \\
\hline FOLLOW HOSPITAL CIRCUIT +/- & $x$ & $x$ & $x$ & $x$ \\
\hline
\end{tabular}

TABLE 2. Summary of discontent plan.

\begin{tabular}{|c|c|c|c|c|}
\hline & $\begin{array}{l}\text { SPECIAL EXAMS } \\
\text { UNIT }\end{array}$ & $\begin{array}{l}\text { OBSTETRICS AND } \\
\text { GYNECOLOGY UNIT }\end{array}$ & $\begin{array}{l}\text { HEMODYNAMICS AND } \\
\text { INTERVENTION RADIOLOGY UNIT }\end{array}$ & $\begin{array}{l}\text { NMR/ CT } \\
\text { UNIT }\end{array}$ \\
\hline $\begin{array}{l}\text { URGENT AND ELECTIVE } \\
\text { PROCEDURES }\end{array}$ & $x$ & $x$ & $x$ & $x$ \\
\hline TEST 48H BEFORE PROCEDURE & $x$ & $x$ & $x$ & $x$ \\
\hline $\begin{array}{l}\text { URGENT, TEST IN } 6 \mathrm{H} \\
\text { BEFORE PROCEDURE }\end{array}$ & $x$ & $x$ & $x$ & $x$ \\
\hline EMERGENT SUCHAS COVID + & $x$ & $x$ & $x$ & $x$ \\
\hline PPE FOR ALL & $x$ & $x$ & $x$ & $x$ \\
\hline DISINFECTION & $x$ & $x$ & $x$ & $x$ \\
\hline FOLLOW HOSPITAL CIRCUIT +/- & $x$ & $x$ & $x$ & $x$ \\
\hline
\end{tabular}


PPE, care should also be taken not to contact contaminated materials.

Another highlight of our experience refers to the screening in all patient's admission, using a questionnaire for suspected SARS-CoV-2 infection and a RT- PCR test for SARS-CoV-2 in all patients proposed for surgery or any procedure with anaesthesia. Although the authors have performed few surgeries on confirmed COVID-19 patients, potential cases in the near future are anticipated if a potential second wave appears. The authors describe some of the thought processes and difficulties they experienced to potentially help others who may find themselves in a similar situation.

\section{CONCLUSION}

Portugal had a high sense of alert and readiness to take whatever measures to control COVID-19 spread. Portugal has imposed a number of extreme measures on social movement, social and religious gathering, travel and businesses, way before the number of cases reached 600 cases. It is expected that Portugal would have a flatter curve than many other countries and may be able to control the pandemic quicker than others. Hospitals were forced to implement dynamic measures that included the modification of infrastructure and processes, management of staff and patients, infection prevention strategies and clinical recommendations. The anesthesiology "stage" is a complex environment with multiple stakeholders including anaesthesiologists, surgeons, nurses, operating room attendants and technicians. Therefore, it can be a challenge to align the interests and concerns of all parties. Nevertheless, we believe we described measures to optimize the quality of care provided to COVID-19 patients and to reduce the risk of viral transmission to other patients or healthcare workers. We hope that this report will help other hospitals to prepare for future COVID-19 outbreaks and infection control in unexpected conditions.

\section{DETAILS OF AUTHOR'S CONTRIBUTION}

All authors give substantial contribution to the study.

AF, AL, CM, MF, PS, RO, SG: Study conception and design and revising it critically for important intellectual content.

AF: final approval to be published.

\section{ACKNOWLEDGMENT/ AGRADECIMENTO}

The authors thank all healthcare workers in the Hospital CUF Porto operating theatre for their ideas, suggestions, excellent work, accountability, availability and competence.

\section{ETHICAL DISCLOSURES}

CONFLICTS OF INTEREST: The authors have no conflicts of interest to declare.

FINANCING SUPPORT: This work has not received any contribution, grant or scholarship.

PROVENANCE AND PEER REVIEW: Not commissioned; externally peer reviewed.

\section{RESPONSABILIDADES ÉTICAS}

CONFLITOS DE INTERESSE: Os autores declaram não possuir conflitos de interesse.

SUPORTE FINANCEIRO: O presente trabalho não foi suportado por nenhum subsídio ou bolsa.

PROVENIÊNCIA E REVISÃO POR PARES: Não comissionado; revisão externa por pares.

\section{REFERENCES}

1. Wong J, Goh QY, Tan Z, Lie SA, Tay YC, et al. Preparing for a COVID-19 pandemic: a review of operating room outbreak response measures in a large tertiary hospital in Singapore. Can J Anaesth. 2020;67:732-45.doi: 10.1007/s12630-02001620-9.

2. Coronavirus-19 disease. Uptodate [May 2020] Available from: https://www.uptodate.com/contents/coronavirus-disease-2019-COVID-19-epidemiology-virology-and-prevention.

3. Greenland JR, Michelow MD, Wang L, London MJ. COVID-19 Infection: Implications for Perioperative and Critical Care Physicians. Anesthesiology. 2020;132:1346-61. doi: 10.1097/ ALN.0000000000003303.

4. Meng L, Qiu H, Wan L, Ai Y, Xue Z, Guo Q, et al. Intubation and Ventilation amid the COVID-19 Outbreak: Wuhan's Experience. Anesthesiology. 2020;132:1317-32. doi: 10.1097/ ALN.0000000000003296.

5. Wong WY, Kong YC, See JJ, Kan RK, Lim MP, Chen Q, et al. Anaesthetic management of patients with COVID-19: infection prevention and control measures in the operating theatre. Br J Anaesth. 2020 (in press). doi: 10.1016/j.bja.2020.04.014.

6. Airway Management Guidance. RCOA, 14 March 2020.[May 2020] Available from: https://rcoa.ac.uk/safe-airway-management-patient-COVID-19.

7. EAMS Airway Management. [May 2020] Available from: Available at https://www.eamshq.net/images/POSTER_EAMS_Rev._1.2.pdf.

8. Chow TT, Kwan A, Lin Z, Bai W. Conversion of operating theatre from positive to negative pressure environment. J Hosp Infect. 2006;64:371-8. doi:10.1016/j.jhin.2006.07.020. 
9. Sehulster L, Chinn RY; CDC; HICPAC. Guidelines for environmental infection control in health-care facilities. Recommendations of CDC and the Healthcare Infection Control Practices Advisory Committee (HICPAC). MMWR Recomm Rep. 2003;52:1-42.

10. Direção Geral Saúde. Prevenção e Controlo de Infeção por SARS - CoV-2 (COVID-19): Equipamentos de Proteção Individual (EPI). Norma 007/2020 1-7 82020). Lisboa: DGS; 2020. 